\section{A) Check for updates}

Cite this: Food Funct., 2018, 9, 1692

\title{
Effect of adding oat bran to instant oatmeal on glycaemic response in humans - a study to establish the minimum effective dose of oat $\beta$-glucan $\dagger$
}

\author{
Thomas M. S. Wolever, (D) *a Alexandra L. Jenkins, ${ }^{a}$ Kevin Prudence, ${ }^{b}$ \\ Jodee Johnson, ${ }^{\mathrm{c}}$ Ruedi Duss, ${ }^{\mathrm{b}}$ YiFang $\mathrm{Chu}^{\mathrm{c}}$ and Robert E. Steinert ${ }^{\mathrm{b}, \mathrm{d}}$
}

Reducing the glycaemic response to carbohydrate-containing foods may have desirable physiological effects for many people. Here, we aimed to determine the minimum amount of oat $\beta$-glucan from oatbran which, when added to instant-oatmeal, would reduce the glycaemic response by $20 \%$ compared to a $\beta$-glucan-free cereal. Therefore, the glycaemic responses elicited by one serving $(27 \mathrm{~g})$ instant-oatmeal plus $247 \mathrm{~g} 2 \%$ milk (IO, $28 \mathrm{~g}$ available-carbohydrate, $1.2 \mathrm{~g} \beta$-glucan) or $1 \mathrm{O}$ plus $0.2,0.4,0.8 \mathrm{or} 1.6 \mathrm{~g}$ oat $\beta$-glucan (OBG) from oat-bran, or an available-carbohydrate matched portion of cream of rice (CR) plus milk were measured over $2 \mathrm{~h}$ in $n=40$ subjects using a randomized, cross-over design. The primary endpoint was incremental area under the curve (iAUC). The secondary endpoint was glucose peak-rise. The results showed that grams OBG consumed were significantly correlated with mean iAUC $(p=0.009)$ and with mean glucose peak-rise ( $p=0.002$ ). Each gram of OBG reduced iAUC by $7 \%$ and peak-rise by $15 \%$. Thus, to achieve $a \geq 20 \%$ reduction in iAUC relative to CR, $1.6 \mathrm{~g} \mathrm{OBG}$ had to be added to $1 \mathrm{O}$ (74 $\pm 7 \mathrm{vs} .93$ $\pm 6 \mathrm{mmol} \mathrm{min} \mathrm{L}^{-1}, p<0.05$ ), but, to achieve a $20 \%$ reduction in peak-rise, only $0.4 \mathrm{~g}$ OBG was required $(2.00 \pm 0.1 \mathrm{vs} .2 .40 \pm 0.1 \mathrm{mmol}, \mathrm{p}<0.05)$. We conclude that adding OBG to IO flattened postprandial glycaemic responses in a dose-dependent fashion; $1.6 \mathrm{~g}$ OBG was required to reduce $\mathrm{i} A \cup \mathrm{C}$ by $\geq 20 \%$ versus $C R$, but a $20 \%$ reduction in peak-rise required only $0.4 \mathrm{~g}$. The greater effect of $O G B$ on peak-rise than iAUC presumably reflects the way viscous dietary fibres modulate glucose absorption kinetics.

Received 9th November 2017, Accepted 26th January 2018 DOI: 10.1039/c7fo01768e rsc.li/food-function
Oat $\beta$-glucan is a highly viscous mixed-linkage $\beta$-glucan ${ }^{6}$ thought to play a key role in reducing postprandial glycaemic and insulinaemic responses ${ }^{7,8}$ by increasing the viscosity of the contents of the upper gut, ${ }^{7,8}$ which, in turn, slows gastric emptying, may reduce the rate of starch digestion and increase the thickness of the so-called unstirred water layer in the small intestine, thus delaying the absorption of carbohydrates. ${ }^{9}$ Most, ${ }^{7,10-22}$ but not all ${ }^{23-25}$ studies have demonstrated that the addition of high molecular-weight oat $\beta$-glucan to test-meals reduces glycaemic responses in human subjects. Thus, the addition of a small amount of oat $\beta$-glucan to instant oatmeal may further reduce its glycaemic impact.

The objective of this study was to determine the minimum amount of oat $\beta$-glucan which needs to be added to a $27 \mathrm{~g}$ serving of instant oatmeal (which already contains $1.2 \mathrm{~g}$ $\beta$-glucan) in order to elicit a $20 \%$ lower incremental area under the blood glucose response curve (iAUC) compared to a $\beta$-glucan-free control cereal. We previously showed that consuming $13.6 \mathrm{~g}$ of oat bran containing $2.6 \mathrm{~g}$ of oat $\beta$-glucan mixed into water just before consuming $110 \mathrm{~g}$ white bread (50 g available carbohydrate) reduced glucose iAUC by about 
Table 1 Nutrient content of test meals

\begin{tabular}{|c|c|c|c|c|c|c|c|c|c|}
\hline \multirow[b]{2}{*}{ Test-meal $^{a}$} & \multirow[b]{2}{*}{ Abbr } & \multirow[b]{2}{*}{ Weight (g) } & \multirow[b]{2}{*}{ Energy (kcal) } & \multirow[b]{2}{*}{ Protein (g) } & \multirow[b]{2}{*}{ Fat $(g)$} & \multicolumn{4}{|c|}{ Carbohydrate $^{b}(\mathrm{~g})$} \\
\hline & & & & & & Total & Fibre & BG & Avail \\
\hline Cream of rice & $\mathrm{CR}$ & 20.0 & 71 & 1.3 & 0 & 16.0 & 0 & 0 & 16.0 \\
\hline Instant oatmeal & IO & 27.0 & 104 & 3.6 & 1.9 & 18.3 & 2.6 & 1.17 & 15.7 \\
\hline Instant oatmeal plus $0.72 \mathrm{~g} \mathrm{OB}$ & $\mathrm{IO}+0.2$ & 27.72 & 106 & 3.8 & 1.9 & 18.7 & 2.77 & 1.37 & 15.8 \\
\hline Instant oatmeal plus $1.43 \mathrm{~g} \mathrm{OB}$ & $\mathrm{IO}+0.4$ & 28.43 & 108 & 3.9 & 2.0 & 19.0 & 2.94 & 1.57 & 15.8 \\
\hline Instant oatmeal plus $2.86 \mathrm{~g} \mathrm{OB}$ & $\mathrm{IO}+0.8$ & 29.86 & 112 & 4.3 & 2.0 & 19.8 & 3.28 & 1.97 & 16.0 \\
\hline Instant oatmeal plus $5.72 \mathrm{~g} \mathrm{OB}$ & $\mathrm{IO}+1.6$ & 32.72 & 120 & 4.9 & 2.2 & 21.3 & 3.97 & 2.77 & 16.2 \\
\hline 2\% Milk $(240 \mathrm{ml})$ & - & 247 & 124 & 8.2 & 4.9 & 11.9 & 0.0 & 0.0 & 11.9 \\
\hline
\end{tabular}

${ }^{a} \mathrm{OB}=$ oat bran (OatWell $®$ ). $2 \%$ milk consumed with every test-meal. ${ }^{b} \mathrm{BG}=$ oat $\beta$-glucan; Avail = available carbohydrate.

$10 \%{ }^{26}$ This dose of oat $\beta$-glucan is equivalent to adding $1.5 \mathrm{~g}$ to a $27 \mathrm{~g}$ serving of instant oats plus $247 \mathrm{~g} 2 \%$ milk (a total of $28 \mathrm{~g}$ of available carbohydrate). However, since $27 \mathrm{~g}$ instant oats already contains $1.2 \mathrm{~g}$ oat $\beta$-glucan and since soluble viscous fibres more effectively reduced glycaemic response when mixed into a food than when taken before eating, ${ }^{27,28}$ we hypothesized that adding as little as $0.4 \mathrm{~g}$ of oat $\beta$-glucan to a serving of instant oats would elicit a glucose iAUC at least $20 \%$ less than that elicited by a $\beta$-glucan-free control cereal.

\section{Materials and methods}

\section{Subjects and study design}

Male and non-pregnant, non-lactating females with fasting whole blood glucose $<6.3 \mathrm{mmol} \mathrm{L}^{-1}$ (corresponding to plasma glucose $<7.0 \mathrm{mmol} \mathrm{L}^{-1}$ ), aged $18-75$ years and with body mass index (BMI) between 20.0 and $34.9 \mathrm{~kg} \mathrm{~m}^{-2}$ were eligible to participate. They were recruited from the pool of subjects at who had previously participated in studies at GI Labs and had given permission to be contacted to be recruited for future studies. The study was performed according to Good Clinical Practice and the Declaration of Helsinki. Ethical approval was obtained from the Western Institutional Review Board and the study was registered as a clinical trial with clinical trials.gov (registration number NCT02818452). Written informed consent was obtained from all participants.

Each participant underwent 6 treatments on separate days using a randomized, cross-over design. On each test day, subjects came to the laboratory in the morning after a 10-14 hours overnight fast. Two fasting blood samples $(t=$ $-5 \mathrm{~min}$ and $t=0 \mathrm{~min}$ ) were obtained by finger-prick. After the second fasting blood sample $(t=0 \mathrm{~min})$, a test-meal was consumed. Further blood samples were obtained at 15, 30, 45, 60, 90 and 120 minutes after starting to eat. Participants remained seated quietly during the 2 hours of the test, and, after the last blood sample had been obtained, were offered a snack and then permitted to leave.

The test-meals consisted of a $\beta$-glucan free cereal, cream of rice cereal (B\&G Foods, Inc., Parsippany, NJ) (CR), instant oatmeal containing $1.2 \mathrm{~g}$ oat $\beta$-glucan (Quaker Oats, Cedar Rapids, IA) alone (IO), or IO plus $0.2,0.4,0.8$ or $1.6 \mathrm{~g}$ of additional oat $\beta$-glucan from an oat bran (OatWell®, DSM Nutritional Products, AG, Basel, Switzerland). The composition of each test-meal is shown in Table 1.

Test-meals were provided in single serve sachets labelled with a code and were prepared by emptying a sachet into a microwave-safe bowl, stirring in $200 \mathrm{ml}$ cold tap-water and heating in a microwave oven on high for $2 \mathrm{~min}$. Cereals were then stirred, allowed to sit for $1 \mathrm{~min}$ and served with $240 \mathrm{ml}$ cold $2 \%$ milk as a beverage.

\section{Randomization and blinding}

The 6 treatments were arranged in 6 orders such that each treatment was first, second, third, fourth, fifth and sixth only once. These six orders were grouped into blocks of 6 or 12 and the order of each block randomized using the RAND( ) function on an excel spreadsheet by a person not involved in scheduling subject visits. A total of 48 such orders were created and assigned to subjects in the order they attended for the first visit after being recruited.

The test-meals were provided in opaque sachets labeled with codes, which were kept by PepsiCo, Inc. However, the comparator cereal (CR) had a different appearance than the oat cereals. Therefore, the study was considered to be singleblind with respect to the comparisons between the comparator and oatmeal cereals (the individuals measuring the glucose responses and cleaning the database were unaware of the treatment assignments); but double-blind with respect to the doses of oat bran, since all the oatmeal test-meals had a similar appearance. After the database had been cleaned by a blinded individual, a copy of the locked database was sent to PepsiCo; and then the codes were broken.

\section{Biochemical analysis}

Blood samples (2-3 drops each) were collected into $5 \mathrm{~mL}$ tubes containing $\sim 500 \mu \mathrm{g}$ sodium fluoride and $400 \mu \mathrm{g}$ potassium oxalate. The samples were mixed and refrigerated immediately during the testing session. After completion of the test session, samples were stored at $-20{ }^{\circ} \mathrm{C}$ prior to glucose analysis. Blood glucose analysis, using an automatic glucose analyzer (Model 2300, Yellow Springs Instruments, OH), took place within five days of collection. In the 218 fasting samples for which duplicates could be measured, mean \pm SD glucose 
was $4.37 \pm 0.0505 \mathrm{mmol} \mathrm{L}^{-1}$ yielding an analytical coefficient of variation of $1.2 \%$.

\section{Viscosity analysis}

The preparation procedure for the test meal was adapted to the Rapid Visco Analyser (RVA 4500, Perten Instruments, Hägersten, Sweden) equipped with Thermocline for window TCW3 software. ${ }^{29}$ Dry test meal samples were reduced in particle size by milling in a lab mill (IKA Works Inc., Willmington, DE) and sieving though a $630 \mathrm{~nm}$ sieve; $X / 8 \mathrm{~g}$ of each milled sample ( $X=$ the total weight of sample given in Table 1) was added to $25 \mathrm{ml}$ deionized water in the RVA container; after the stirring paddle was placed into the container and mounted onto the RVA, the cycle was started. The protocol was based on the recommendation of testing oat quality ${ }^{30}$ with the temperature being raised by $16.6{ }^{\circ} \mathrm{C} \min ^{-1}$ to $80{ }^{\circ} \mathrm{C}$, followed by a $1 \mathrm{~min}$ lag-time for equilibration, cooling by $5{ }^{\circ} \mathrm{C} \mathrm{min}^{-1}$ to $25{ }^{\circ} \mathrm{C}$ and 2 min equilibration time at $25{ }^{\circ} \mathrm{C}$ with constant stirrer speed of $100 \mathrm{rpm}$ (total cycle time $18 \mathrm{~min}$, read-out final $\mathrm{mPa}$ ). All samples were measured in triplicate.

\section{Calculations}

Data were entered into a spreadsheet by 2 different individuals and the values compared to assure accurate transcription. Incremental areas under the blood glucose response curve (iAUC), ignoring area below fasting, were calculated using the trapezoid rule. ${ }^{31,32}$ Peak-rise was defined as the maximum glucose concentration during the 2-hour test minus the fasting concentration. Fasting glucose was taken to be the mean of the concentrations in the 2 fasting samples.

Glucose was measured in the 0 min fasting sample 2 times and the data used to determine the SD of the analytical variation as follows:

$$
\mathrm{SD}=\sqrt{ }\left(\sum d^{2} / n\right)
$$

\section{Statistical analysis}

Glucose iAUC and peak-rise were analysed using repeatedmeasures analysis of variance (RMANOVA) examining for the main effect of test-meal. Blood glucose concentrations and increments were analyzed using RMANOVA examining the main effects of time and test-meal and the time $\times$ test-meal interaction. After demonstrating significant time $\times$ test-meal interactions for glucose concentrations $\left(F_{(35,1365)}=10.81, p=\right.$ $\left.3.0 \times 10^{-51}\right)$ and increments $\left(F_{(30,1170)}=11.40, p=5.5 \times 10^{-47}\right)$, indicating that the effect of test-meal varied by time, the glucose concentrations and increments at each time point were analyzed using RMANOVA examining for the main effect of test-meal. After demonstration of significant heterogeneity, the individual means were compared to the control (CR) using Dunnett's test to adjust for multiple comparisons (GraphPad Prism, GraphPad Software, La Jolla, CA). Results for meal viscosity were analyzed similarly except that individual means were compared to each other using Tukey's test to adjust for multiple comparisons. The criterion for statistical significance was a 2-tailed $p<0.05$. Pearson's correlation coefficients were calculated using the method of least squares (GraphPad Prism).

\section{Power analysis}

From a previous study ${ }^{33}$ the SD of the differences in iAUC between oatmeal and cream of rice was $37.6 \mathrm{mmol} \mathrm{min} \mathrm{L}^{-1}$ for portions of test-meals containing $18 \mathrm{~g}$ available carbohydrate (avCHO), $26.5 \mathrm{mmol} \mathrm{min} \mathrm{L}^{-1}$ for portions containing $24 \mathrm{~g}$ avCHO and $51.7 \mathrm{mmol} \mathrm{min}^{-1}$ for portions containing $36 \mathrm{~g}$ avCHO. In the present study, the test-meals contained $28 \mathrm{~g}$ avCHO, therefore, the SD of the differences in iAUC was estimated, conservatively, to be $40 \mathrm{mmol} \mathrm{min} \mathrm{L}^{-1}$. Using this $\mathrm{SD}$, and, based on the $t$-distribution, there is $80 \%$ power with $n=$ 40 subjects to detect a difference in iAUC of $18.2 \mathrm{mmol} \mathrm{min} \mathrm{L}^{-1}$, which is approximately $15-20 \%$ of the iAUC expected for the comparator test-meal.

\section{Results}

\section{Test-meal viscosity}

The viscosity of IO was significantly greater than that of CR, and viscosity increased exponentially as the amount of OBG in the test meal increased $\left(r^{2}=0.994\right.$, ESI Fig. $\left.1 \dagger\right)$. The viscosity of each test-meal was significantly different from that of every other test meal (Table 2).

\section{Subjects}

We recruited 41 subjects; one dropped-out for personal reasons after completing 1 test and 40 (22 males, 18 females),

Table 2 Viscosity of the test meals and their glycaemic responses

\begin{tabular}{|c|c|c|c|c|}
\hline Test-meal & Abbr & Final viscosity ${ }^{a}$ (mPa s) & 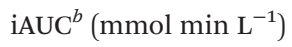 & Peak-rise $^{b}\left(\mathrm{mmol} \mathrm{L}^{-1}\right)$ \\
\hline Cream of rice & CR & $178 \pm 4^{\mathrm{a}}$ & $92.9 \pm 5.8$ & $2.44 \pm 0.12$ \\
\hline Instant oatmeal & IO & $1500 \pm 27^{\mathrm{b}}$ & $87.5 \pm 6.7$ & $2.18 \pm 0.12^{*}$ \\
\hline Instant oatmeal plus $0.72 \mathrm{~g} \mathrm{OB}^{c}$ & $\mathrm{IO}+0.2$ & $2280 \pm 10^{c}$ & $81.3 \pm 6.6$ & $2.13 \pm 0.09^{*}$ \\
\hline Instant oatmeal plus $1.43 \mathrm{~g} \mathrm{OB}^{c}$ & $\mathrm{IO}+0.4$ & $3020 \pm 8^{\mathrm{d}}$ & $86.1 \pm 6.9$ & $2.00 \pm 0.11^{*}$ \\
\hline Instant oatmeal plus $2.86 \mathrm{~g} \mathrm{OB}^{c}$ & $\mathrm{IO}+0.8$ & $5220 \pm 68^{\mathrm{e}}$ & $81.1 \pm 5.8$ & $1.75 \pm 0.09^{*}$ \\
\hline Instant oatmeal plus $5.72 \mathrm{~g} \mathrm{OB}^{c}$ & $\mathrm{IO}+1.6$ & $11930 \pm 240^{f}$ & $73.8 \pm 6.6^{*}$ & $1.45 \pm 0.08^{*}$ \\
\hline
\end{tabular}

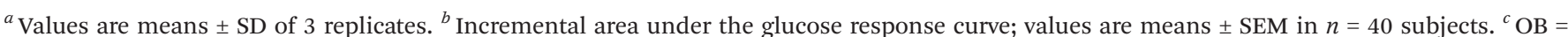

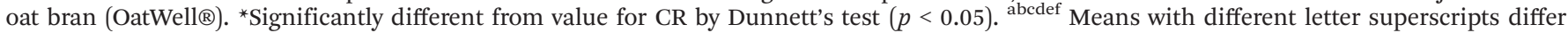
significantly $(p<0.05)$ by Tukey's test. 


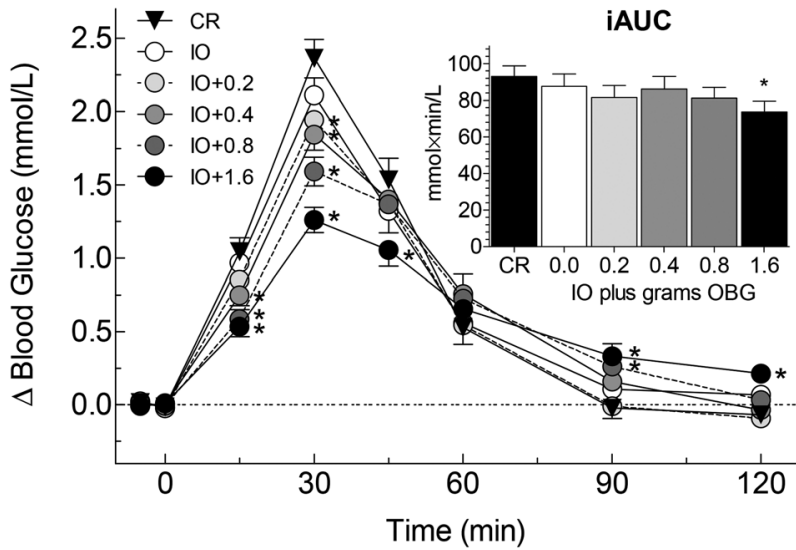

Fig. 1 Glycaemic responses elicited by the test meals. Main panel: Blood glucose increments after the test-meals; values are means \pm SEM $(n=40)$. CR, Cream of Rice; IO, Instant Oatmeal; $10+0.210$ plus $0.2 \mathrm{~g}$ oat $\beta$-glucan; $10+0.4$, 10 plus $0.4 \mathrm{~g}$ oat $\beta$-glucan; $10+0.8$, 10 plus $0.8 \mathrm{~g}$ oat $\beta$-glucan; $10+1.6,10$ plus $1.6 \mathrm{~g}$ oat $\beta$-glucan. Inset: Incremental areas under the curve (iAUC, means \pm SEM, $n=40$ ). OBG, oat $\beta$-glucan. * Significantly different from CR (Dunnett's test, $p<0.05$ ).

aged 18 to 57 years (mean \pm SD, $31.5 \pm 11.0$ year) with BMI from 20.1 to $31.5 \mathrm{~kg} \mathrm{~m}^{-2}$ (mean $\pm \mathrm{SD} 25.1 \pm 3.0 \mathrm{~kg} \mathrm{~m}^{-2}$ ) completed the study. All subjects had fasting glucose $<5.7 \mathrm{mmol} \mathrm{L}^{-1}$ $\left(103 \mathrm{mg} \mathrm{dL} \mathrm{dL}^{-1}\right.$ ). Descriptions of participants' use of sup- plements/drugs, minor protocol violation, and how missing values were handled are given in the ESI. $\dagger$

\section{Glycaemic responses}

There was significant heterogeneity among treatments for the primary endpoint, iAUC, $\left(F_{(5,195)}=2.51, p=0.032\right)$ with iAUC after IO +1.6 being significantly less than that after $\mathrm{CR}$ (Table 2, Fig. 1 inset). There was a significant correlation between iAUC and grams $\beta$-glucan in the test-meal $(r=-0.920$, $p=0.009)$ and between iAUC and $\log$ (viscosity) $(r=-0.918, p=$ 0.010) (Fig. 2). Relative to the iAUC after CR, each gram of oat $\beta$-glucan in the test-meal reduced iAUC by $7.0 \%$. Thus, the addition of $1.6 \mathrm{~g}$ oat $\beta$-glucan was required to obtain a $20 \%$ reduction in iAUC.

There was no significant difference in fasting glucose among the 6 treatments. Incremental blood glucose responses are shown in Fig. 1. There was significant heterogeneity of mean blood glucose increments among treatments at 15 $\left(F_{(5,195)}=13.63, p=2.1 \times 10^{-11}\right), 30\left(F_{(5,195)}=27.98, p=2.5 \times\right.$ $\left.10^{-21}\right), 45\left(F_{(5,195)}=3.45, p=0.0052\right), 90\left(F_{(5,195)}=6.32, p=\right.$ $0.000018)$ and $120 \min \left(F_{(5,195)}=7.34, p=2.5 \times 10^{-6}\right)$. The significant differences between the means of the oat treatments and the control are shown in Fig. 1.

There was significant heterogeneity among treatments for peak-rise $\left(F_{(5,195)}=28.81, p=7.9 \times 10^{-22}\right)$ with peak-rise after CR being significantly greater than that after every other test
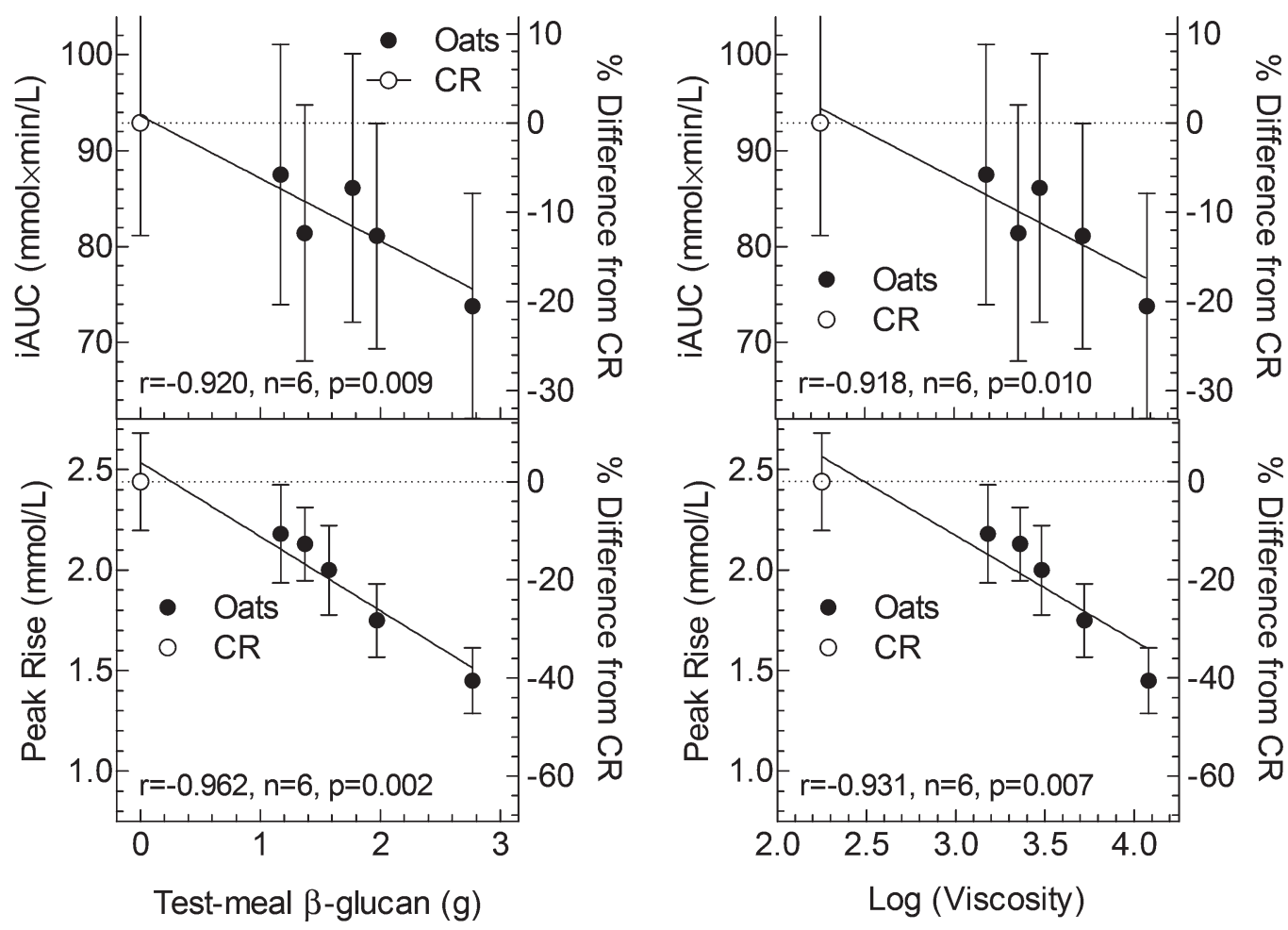

Fig. 2 Relationships between test-meal $\beta$-glucan content/viscosity and glycaemic response. Relationships between test-meal $\beta$-glucan content (left graphs) and log (test-meal viscosity) (right graphs) and incremental area under the curve (iAUC, top graphs) or peak-rise (bottom graphs). The left $y$-axes are absolute values; the right $y$-axes show the $\%$ difference from control (CR). Points are means and $95 \%$ confidence intervals in $n=40$ subjects, solid lines are regressions. Oats $=$ instant oats with or without added oat $\beta$-glucan; $C R=$ cream of rice. 
meal (Table 2). There was a significant correlation between peak-rise and grams $\beta$-glucan in the test-meal $(r=-0.962$, $n=6, p=0.002$ ) and between peak-rise and $\log$ (viscosity) ( $r=$ $-0.931, p=0.007$ ) (Fig. 2). Relative to CR, each gram of oat $\beta$-glucan in the test-meal reduced peak-rise by $15 \%$, thus, the addition of $0.4 \mathrm{~g}$ oat $\beta$-glucan to IO resulted in a $20 \%$ reduction in peak-rise.

\section{Discussion}

The results show that adding graded amounts of oat $\beta$-glucan from oat bran to instant oats resulted in dose-dependent reductions in both glucose iAUC and peak-rise. Mean iAUC after IO plus $1.6 \mathrm{~g}$ oat $\beta$-glucan ( $\mathrm{IO}+1.6)$ was $20 \%$ less than that after CR $(p=0.03)$; although mean glucose iAUC after IO +0.2 , IO +0.4 and IO +0.8 were 6 to $13 \%$ less than that after $\mathrm{CR}$, none of the differences was statistically significant. However, every oat containing test meal elicited a significantly lower peak-rise than $\mathrm{CR}$, with the percentage reductions ranging from $11 \%$ after IO alone to $41 \%$ after $\mathrm{IO}+1.6$.

Our results are novel in demonstrating a significant doseresponse effect of low amounts of oat $\beta$-glucan on glycaemic response. According to the scientific opinion of the European Food Safety Authority (EFSA), $4 \mathrm{~g}$ of oat $\beta$-glucan per $30 \mathrm{~g}$ of available carbohydrates (i.e. $0.133 \mathrm{~g}$ OBG per $\mathrm{g}$ avCHO) is required to obtain a reliable effect on glycaemic response; ${ }^{34}$ thus, amounts $<0.133 \mathrm{~g}$ OBG per $\mathrm{g}$ avCHO would be considered to be too low. Upon reviewing the literature, we found 17 studies $^{7,10-25}$ reporting a total of 29 comparisons of the effect of high molecular weight oat $\beta$-glucan (OBG) on glucose iAUC (or providing data from which to calculate iAUC). Glycaemic response was reduced in all 7 of the comparisons using high amounts of oat $\beta$-glucan $(\geq 0.133 \mathrm{~g}$ OBG per $\mathrm{g}$ avCHO), and amongst these 7 there was a significant doseresponse with each $0.1 \mathrm{~g}$ OBG per $\mathrm{g}$ avCHO reducing glucose AUC by $22 \%$ (Fig. 3). However, in 8 (35\%) of the 22 comparisons testing a low amount of oat $\beta$-glucan (i.e. $<0.133 \mathrm{~g}$ OBG per $\mathrm{g}$ avCHO) there was no effect on glycaemic response and, in all 22 comparisons, no trend for a dose-response effect (Fig. 3). In the present study we tested low amounts of oat $\beta$-glucan ( 0.04 to 0.1 gOBG per $g$ avCHO) and found a significant dose-response effect on glucose iAUC with each 0.1 gOBG per $\mathrm{g}$ avCHO reducing iAUC by $20 \%$, an effect equivalent to that seen in the 7 studies using higher amounts of oat $\beta$-glucan.

iAUC was chosen as the primary endpoint because it is generally considered to be a valid and reliable way to express glycaemic responses. A $20 \%$ reduction in glucose iAUC has been suggested by Health Canada to be the minimum reduction required to support a claim related to a reduced glycaemic response. ${ }^{35}$ Thus, we were interested to know how much, if any, oat $\beta$-glucan would have to be added to IO to result in a $\geq 20 \%$ reduction in glucose iAUC compared to an avCHO matched portion of CR; the present results show that at least $1.6 \mathrm{~g}$ oat $\beta$-glucan is needed to achieve this target (Fig. 2).

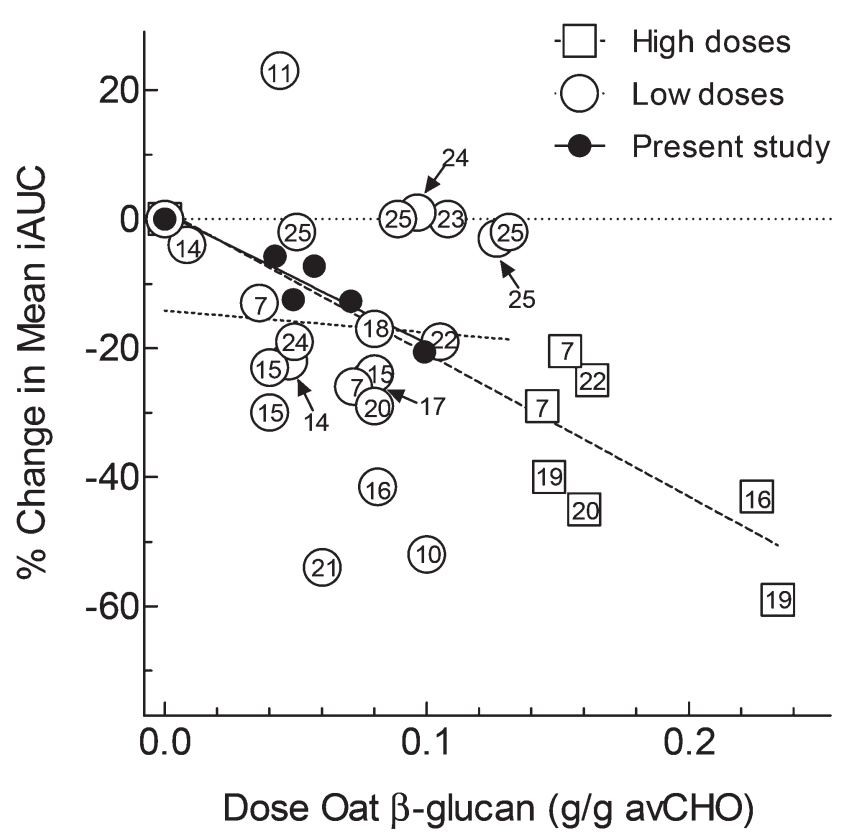

Fig. 3 Present results compared to those in the literature. Relationship between dose of oat $\beta$-glucan ( $\mathrm{g} / \mathrm{g}$ available carbohydrate (avCHO) in the test-meal) and the \% change in glycaemic response (incremental area under the curve, iAUC) for studies in the literature using high doses of oat $\beta$-glucan ( $\geq 0.133 \mathrm{~g} / \mathrm{g}$ avCHO, open squares), low doses of oat $\beta$-glucan $(<0.133 \mathrm{~g} / \mathrm{g}$ avCHO, open circles) and the present study (closed circles). The number within each symbol is the study number in the list of references (arrows identify circles partly or completely hidden). Dashed line: Regression line for high doses $(r=-0.491, p=0.003)$; dotted line: regression line for low dose studies $(r=0.064$, ns); solid line: regression line for present study $(r=-0.938, p=0.006)$. See graphical abstract for colour figure.

EFSA $^{34}$ does not define a minimum \% reduction in glucose iAUC and, therefore, it could be argued that a consistent 10\% reduction in postprandial glycaemic response may be physiologically relevant. The present results suggest that the addition of $0.3 \mathrm{~g}$ of oat $\beta$-glucan to IO would have such an effect, however, post-hoc power analysis suggests that over 300 subjects would be needed to have $80 \%$ power to detect a significant $10 \%$ reduction in glucose iAUC.

In contrast to the few significant differences and relatively small effect sizes for glucose iAUC we observed, the average effect-size for glucose peak-rise was nearly twice that for iAUC (on a percentage basis) and the reduction in peak-rise which could be detected with statistical significance was about half that for iAUC (Fig. 2). This is in contrast to the typical situation in which \% reductions in peak rise are similar to the \% reductions in iAUC ${ }^{36,37}$ or glycemic index. ${ }^{38}$ The smaller relative effect size of oat $\beta$-glucan on iAUC compared to its effect on peak-rise as seen here is consistent with the results of other studies of oat $\beta$-glucan. ${ }^{20}$ We hypothesize this effect is due to an ability of viscous fibre to reduce the rate and prolong the time of carbohydrate absorption from the small intestine ${ }^{9}$ resulting in a reduced peak of blood glucose and a delayed return to baseline. In normal subjects, the slower return to 
baseline after oat $\beta$-glucan-enriched test meals leads to a higher iAUC during the period from 1-2 $\mathrm{h}$ after eating which partly offsets the reduction in iAUC seen from $0-1 \mathrm{~h}$. This effect can result in a total iAUC value $(0-2 \mathrm{~h})$ that is similar to that of the control meal despite having a very different glucose profile, suggesting a potential limitation of focusing on iAUC as the only criterion used to evaluate postprandial glycaemic responses.

Flattening the glucose response by reducing peak-rise reduces postprandial glucose fluctuations, which, in turn, may reduce oxidative stress and inflammation even in the absence of a significant reduction in iAUC. For example, exposing cultured endothelial cells to oscillating glucose concentrations elicits greater apoptosis ${ }^{39}$ and metabolic memory ${ }^{40}$ than does exposing them to a constant high glucose concentration. In patients with type 2 diabetes, a high magnitude of glucose fluctuations was associated with increased oxidative stress independently of overall glycaemic control as assessed by HbA1c. ${ }^{41,42}$ Furthermore, in lean subjects without diabetes it has been demonstrated that test-meals eliciting flatter glycaemic responses were associated with reduced postprandial NFкB activation in white blood cells. ${ }^{43}$ Finally, Esposito et $a l .{ }^{44}$ showed in 644 outpatients with type 2 diabetes that incremental glucose peaks correlated significantly with centralized carotid intima-media thickness, a consolidated surrogate marker of atherosclerosis. The clinical importance of reducing postprandial glucose oscillations, however, warrants further documentation.

We measured final viscosity of the test meal and found that increasing doses of oat $\beta$-glucan significantly increased test meal viscosity. We also found a significant positive correlation between viscosity and effects on glycaemic responses (both for iAUC and peak-rise) suggesting that increases in viscosity and a slowing of gastric emptying are the underlying mechanisms of the observed effects. Indeed, the rate of gastric emptying affects primarily the peak glucose response and oat $\beta$-glucan has been shown to have potent effect on slowing gastric emptying. ${ }^{45,46}$ However, we did not measure gastric emptying, which represent a weakness of the study.

The strengths of this study include the larger number of doses of oat $\beta$-glucan tested than most other studies, and the large number of subjects that provided the statistical power required to detect small differences in iAUC and peak-rise. In addition, because of the wide inclusion/exclusion criteria, the results apply to a large proportion of the population. There were equivalent numbers of subjects who were, respectively, male and female $(n=22$ and 18), lean (BMI < 25) and overweight (BMI $\geq 25)$ and Caucasian and non-Caucasian $(n=18$ and 22) and a reasonably large number of older (age $>40$ ) versus younger (age $\leq 40$ ) subjects. There was no significant difference in response for iAUC or peak rise based on sex, BMI or ethnicity and no effect of age on peak rise (ESI Fig. 2 and $3 \dagger$ ). The only significant difference was that younger subjects had significantly lower iAUC than older subjects after the oatmeal test meals. However, this could have occurred by chance since these post-hoc tests were not corrected for mul- tiple comparisons; with a 5\% chance of success, the chance of 1 success in 8 tries ( 2 endpoints $\times 4$ subject characteristics) is $\sim 28 \%$.

Another weakness is that the commercial oat bran used as a source of oat $\beta$-glucan contains small amounts of protein, fat and available carbohydrate which could confound the results. The effects of oat protein and oat fat on glycemic responses are not known. However, adding up to $30 \mathrm{~g}$ soy protein and fat (corn oil) to $50 \mathrm{~g}$ glucose reduces glycemic responses in a linear fashion, with each gram of protein and fat, respectively, reducing blood glucose peak rise by $1.2 \%$ and $0.6 \%$ and iAUC by $1.3 \%$ and $0.5 \% .{ }^{47}$ On the other hand, available carbohydrate (avCHO) increases the glycemic responses in a nonlinear fashion with the effect of $g$ grams avCHO on iAUC being proportional to $\left(1-e^{-0.0222 \mathrm{~g}}\right) .{ }^{48}$ Adjusting for the effect of the protein and fat contained in the oat bran attenuates the reduction in iAUC relative to Instant Oats alone by a small amount, but this is effect is almost entirely counteracted by the effect of the additional carbohydrate (ESI Fig. $4 \dagger$ ). The dose-response effect of carbohydrate on peak rise is unknown, but the effect of the protein and fat in oat bran is estimated to have had a negligible effect on peak rise (ESI Fig. $4 \dagger$ ).

The lack of insulin measurement is another weakness. However, our aim was not to investigate the mechanism by which oat $\beta$-glucan reduces glycemic responses, but rather to establish the minimum amount required for efficacy. Most studies show that adding oat $\beta$-glucan to test-meals reduces postprandial insulin, even in the absence of a reduction in glucose. $^{25}$ It seems unlikely, therefore, that the reduced glucose responses we observed were due to increased postprandial insulin concentrations.

\section{Conclusions}

It is concluded that adding oat $\beta$-glucan to IO reduces postprandial glycaemic responses in a dose-dependent fashion. Depending on what is considered the critical parameter (iAUC or peak-rise) and what is considered a physiologically relevant reduction, different amounts of $\mathrm{OBG}$ need to be added to achieve the target. For example to achieve a $20 \%$ reduction in iAUC relative to the comparator cereal, CR, $1.6 \mathrm{~g}$ of OBG needs to be added to a serving of IO, in contrast, the results suggest that adding only $0.4 \mathrm{~g}$ OBG reduces the glucose peak-rise by $20 \%$.

\section{Conflicts of interest}

TW, his wife and AJ are owners and employees of Glycemic Index Laboratories, Inc. (GI Labs), a contract research organization; neither they nor GI Labs has any financial interest in any company involved in producing, distributing or marketing foods or food ingredients and, in particular, no financial interest in DSM or PepsiCo. RES, RD and KP are employees of DSM Nutritional Products, Basel, Switzerland; they do not have any 
conflict of interest regarding the publication of this article. JJ and YC are employees of PepsiCo, Inc., Barrington, IL. Disclaimer: The views expressed in this article are those of the authors and do not necessarily reflect the position or policies of PepsiCo, Inc.

\section{Acknowledgements}

We thank Simone Koenig-Grillo for the analysis of viscosity of the test meals. Financial support provided by DSM and Pepsico.

\section{References}

1 S. M. Tosh and Y. Chu, Systematic review of the effect of processing of whole-grain oat cereals on glycaemic responses, Br. J. Nutr., 2015, 114, 1256-1262.

2 D. J. A. Jenkins, T. M. S. Wolever, R. H. Taylor, H. M. Barker, H. Fielden, J. M. Baldwin, A. C. Bowling, H. C. Newman, A. L. Jenkins and D. V. Goff, Glycemic index of foods: a physiological basis for carbohydrate exchange, Am. J. Clin. Nutr., 1981, 34, 362-366.

3 D. J. A. Jenkins, T. M. S. Wolever, A. L. Jenkins, M. J. Thorne, R. Lee, J. Kalmusky, R. Reichert and G. S. Wong, The glycaemic index of foods tested in diabetic patients: a new basis for carbohydrate exchange favouring the use of legumes, Diabetologia, 1983, 24, 257-264.

4 T. M. S. Wolever, L. Katzman-Relle, A. L. Jenkins, V. Vuksan, R. G. Josse and D. J. A. Jenkins, Glycaemic index of 102 complex carbohydrate foods in patients with diabetes, Nutr. Res., 1994, 14, 651-669.

5 T. Wolever, A. Jenkins, J. Campbell, S. Spruill, R. Black, L. Harkness and J.-W. vanKlinken, Differential serving sizes of high $\beta$ glucan oatmeal elicit lower glycemic response than rice cereal, FASEB J., 2015, 29, LB360.

6 P. J. Wood, Oat $\beta$-glucan: structure, location and properties, in Oats: Chemistry and technology, ed. F. H. Webstger, AACC Inc., St Paul, MN, 1986, pp. 121-152.

7 P. J. Wood, J. T. Braaten, F. W. Scott, K. D. Riedel, M. S. Wolynetz and M. W. Collins, Effect of dose and modification of viscous properties of oat gum on plasma glucose and insulin following an oral glucose load, Br. J. Nutr., 1994, 72, 731-743.

8 P. J. Wood, M. U. Beer and G. Butler, Evaluation of role of concentration and molecular weight of oat $\beta$-glucan in determining effect of viscosity on plasma glucose and insulin following an oral glucose load, Br. J. Nutr., 2000, 84, 19-23.

9 D. J. A. Jenkins, T. M. S. Wolever, A. R. Leeds, M. A. Gassull, P. Haisman, J. Dilawari, D. V. Goff, G. L. Metz and K. G. M. Alberti, Dietary fibres, fibre analogues, and glucose tolerance: importance of viscosity, $\mathrm{Br}$. Med. J., 1978, 1, 1392-1394.
10 M. Biörklund, A. van Rees, R. P. Mensink and G. Önning, Changes in serum lipids and postprandial glucose and insulin concentrations after consumption of beverages with $\beta$-glucans from oats or barley: a randomized dose-controlled trial, Eur. J. Clin. Nutr., 2005, 59, 1272-1281.

11 K. C. Maki, R. Galant, P. Samuel, J. Tesser, M. S. Witchger, J. D. Ribaya-Mercado, J. B. Blumberg and J. Geohas, Effects of consuming foods containing oat $\beta$-glucan on blood pressure, carbohydrate metabolism and biomarkers of oxidative stress in men and women with elevated blood pressure, Eur. J. Clin. Nutr., 2007, 61, 786-795.

12 N. Tapola, H. Karvonen, E. Sarkkinen, L. Niskanen and M. Mikola, Glycemic responses of oat bran products in type 2 diabetic patients, Nutr., Metab. Cardiovasc. Dis., 2005, 15, 255-261.

13 M. Kabir, J. M. Oppert, H. Vidal, F. Bruzzo, C. Fiquet and P. Wursch, Four-week low glycemic index breakfast with a modest amount of soluble fibers in type 2 diabetic men, Metabolism, 2002, 51, 819-826.

14 K. M. Behall, D. J. Scholfield, J. Hallfrisch and H. G. M. Liljeberg-Elmstahl, Comparison of both resistant starch and b-glucan improves postprandial plasma glucose and insulin in women, Diabetes Care, 2006, 29, 976-981.

15 H. Mäkeläinen, H. Anttila, J. Sihvonen, R. M. Hietanen, R. Tahvonen, E. Salminen, M. Mikola and T. SontagStrohm, The effect of $\beta$-glucan on the glycemic and insulin index, Eur. J. Clin. Nutr., 2007, 61, 779-785.

16 J. T. Braaten, P. J. Wood, F. W. Scott, K. D. Riedel, L. M. Poste and M. W. Collins, Oat gum, a soluble fiber which lowers glucose and insulin in normal individuals after an oral glucose load: comparison with guar gum, Am. J. Clin. Nutr., 1991, 53, 1425-1430.

17 M. De Angelis, C. G. Rizzello, G. Alfonsi, P. Arnault, S. Cappelle, R. Di Cagno and M. Gobbetti, Use of sourdough lactobacilli and oat fibre to decrease the glycaemic index of white wheat bread, Br. J. Nutr., 2007, 98, 11961205.

18 S. Panahi, A. Ezatagha, F. Temelli, T. Vasanthan and V. Vuksan, b-Glucan from two sources of oat concentrates affect postprandial glycemia in relation to the level of viscosity, J. Am. Coll. Nutr., 2007, 26, 639-644.

19 P. J. Wood, J. T. Braaten, F. W. Scott, D. Riedel and L. M. Poste, Comparisons of viscous properties of oat and guar gum and the effects of these and oat bran on glycemic index, J. Agric. Food Chem., 1990, 38, 753-757.

20 S. M. Tosh, Y. Brummer, T. M. S. Wolever and P. J. Wood, Glycemic response to oat bran muffins treated to vary molecular weight of $\beta$-glucan, Cereal Chem., 2008, 85, 211-217.

21 P. Vitaglione, R. B. Lumaga, A. Stanzione, L. Scalfi and V. Fogliano, $\beta$-glucan-enriched bread reduces energy intake and modifies plasma ghrelin and peptide YY concentrations in the short term, Appetite, 2009, 53, 338-344.

22 A. Regand, Z. Chowdhury, S. M. Tosh, T. M. S. Wolever and P. Wood, The molecular weight, solubility and viscosity of oat beta-glucan affect human glycemic response by modifying starch digestibility, Food Chem., 2011, 129, 297-304. 
23 K. S. Juntunen, L. K. Niskanen, K. H. Liukkonen, K. S. Poutanen, J. J. Holst and H. M. Mykkanen, Postprandial glucose, insulin, and incretin responses to grain products in healthy subjects, Am. J. Clin. Nutr., 2002, $75,254-262$.

24 K. R. Juvonen, M. Salmenkallio-Marttila, M. Lyly, K.-H. Liukkonen, L. Lähteennmäki, D. E. Laaksonen, M. I. Uusitupa, K. H. Herzig, K. S. Poutanen and L. J. Karhunen, Semisolid meal enriched in oat bran decreases plasma glucose and insulin levels, but does not change gastrointestinal peptide responses or short-term appetite in healthy subjects, Nutr., Metab. Cardiovasc. Dis., 2011, 21, 748-756.

25 E. J. Beck, S. M. Tosh, M. J. Batterham, L. C. Tapsell and X.-F. Huang, Oat $\beta$-glucan increases postprandial cholecystokinin levels, decreases insulin response and extends subjective satiety in overweight subjects, Mol. Nutr. Food Res., 2009, 53, 1343-1351.

26 R. E. Steinert, D. Raederstorff and T. M. S. Wolever, Effect of consuming oat bran mixed in water before a meal on glycemic responses in healthy humans - a pilot study, Nutrients, 2016, 8, 524.

27 D. J. Jenkins, R. Nineham, C. Craddock, P. Craig-McFeely, K. Donaldson, T. Leigh and J. Snook, Fibre in diabetes, Lancet, 1979, 1, 434-435.

28 S. Fuessl, T. E. Adrian, A. J. Bacarese-Hamilton and S. R. Bloom, Guar in NIDD: Effect of different modes of administration on plasma glucose and insulin responses to a starch meal, Pract. Diabetes Int., 1986, 3, 258-260.

29 The RVA Handbook, ed. G. B. Crosbie and A. S. Ross, AACC International, St. Paul, MN, 2007.

30 M. Zhou, M. Glennie-Holmes, K. Robards and S. Helliwell, Effects of processing and short-term storage on the pasting characteristics of slurries made from raw and rolled oats, Food Aust., 1999, 51, 251-258.

31 T. M. S. Wolever and D. J. A. Jenkins, The use of the glycemic index in predicting the blood glucose response to mixed meals, Am. J. Clin. Nutr., 1986, 43, 167-172.

32 T. M. S. Wolever, Effect of blood sampling schedule and method calculating the area under the curve on validity and precision of glycaemic index value, Br. J. Nutr., 2004, 91, 295-300.

33 T. M. S. Wolever, B. J. W. van Klinken, S. E. Spruill, A. L. Jenkins, Y. Chu and L. Harkness, Effect of serving size and addition of sugar on the glycemic response elicited by oatmeal: A randomized, cross-over study, Clin. Nutr. ESPEN, 2016, 16, 48-54.

34 EFSA Panel on dietetic Products, Nutrition and Allergies (NDA), Scientific Opinion on the substantiation of health claims related to beta-glcuans from oats and barley and maintenance of normal blood LDL-cholesterol concentrations (ID1236, 1299), increase in satiety leading to a reduction in energy inake (ID 851, 852), reduction in postprandial glycaemic response (ID 821, 824), and "digestive function" (ID 850) pursuant to Article 13(1) of Regulation (EC) No 1924/2006, EFSA J., 2011, 9, 2207.
35 Bureau of Nutritional Sciences, Food Directorate, Health Products and Food Branch, Health Canada, Draft guidance document on food health claims related to the reduction in post-prandial glycaemic response, June, 2013, URL: https:/chfa.ca/images/uploads/2012/08/Post-PrandialGlycaemic-Response-Draft-Guidance.pdf (accessed 17 May, 2017).

36 J. L. Lui, X. Lan-Pidhainy, Y. Brummer, S. M. Tosh, P. J. Wood and T. M. S. Wolever, Effect of blood sampling schedule on the ability to discriminate between postprandial glycemic responses, Nutrition, 2009, 25, 1064-1066.

37 T. M. S. Wolever, J.-W. van Klinken, S. E. Spruill, A. L. Jenkins, Y. Chu and L. Harkness, Effect of serving size and addition of sugar on the glycemic response elicited by oatmeal: A randomized, cross-over study, Clin. Nutr. ESPEN, 2016, 16, 48-54.

38 J. C. Brand-Miller, K. Stockmann, F. Atkinson, P. Petocz and G. Denyer, Glycemic index, postprandial glycemia, and the shape of the curve in healthy subjects: analysis of a database of more than 1000 foods, Am. J. Clin. Nutr., 2009, 89, 97-105.

39 A. Risso, F. Mercuri, L. Quagliaro and A. Ceriello, Intermittent high glucose enhances apoptosis in human umbilical vein endothelial cells in culture, Am. J. Physiol.: Endocrinol. Metab., 2001, 281, E924-E930.

40 B. Schisano, G. Tripathi, K. McGee, P. G. McTernan and A. Ceriello, Glucose oscillations, more than constant high glucose, induce p53 activation and a metabolic memory in human endothelial cells, Diabetologia, 2011, 54, 12191226.

41 L. Monnier, E. Mas, C. Ginet, F. Mechel, L. Villon, J. P. Cristol and C. Colette, Activation of oxidative stress by acute glucose fluctuations compared to sustained chronic hyperglycemic in patients with type 2 diabetes, J. Am. Med. Assoc., 2006, 295, 1681-1687.

42 L. Monnier, C. Colette, E. Mas, F. Michel, J. P. Cristol, C. Boegner and D. R. Owens, Regulation of oxidative stress by glycaemic control: evidence for an independent inhibitory effect of insulin therapy, Diabetologia, 2010, 53, 562-571.

43 S. Dickinson, D. P. Hancock, P. Petocz, A. Ceriello and J. Brand-Miller, High-glycemic index carbohydrate increases nuclear factor-kappaB activation in mononuclear cells of young, lean healthy subjects, Am. J. Clin. Nutr., 2008, 87, 1188-1193.

44 K. Esposito, M. Ciotola, D. Carlen, B. Schisano, L. Sardelli, D. Di Tommaso, L. Misso, F. Saccomanno, A. Ceriello and D. Guigliano, Post-meal glucose peaks at home associate with carotid intima-media thickness in type 2 diabetes, J. Clin. Endocrinol., 2008, 93, 1345-1350.

45 C. S. Marathe, M. Horowitz, L. G. Trahair, J. M. Wishart, M. Bound, K. Lange, C. K. Rayner and K. L. Jones, Relationships of early and late glycemic responses with gastric emptying during an oral glucose tolerance test, J. Clin. Endocrinol. Metab., 2015, 100, 3565-3571.

46 K. R. Juvonen, A. K. Purhonen, M. Salmenkallio-Marttila, L. Lähteenmäki, D. E. Laaksonen, K. H. Herzig, 
M. I. Uusitupa, K. S. Poutanen and L. J. Karhunen, Viscosity of oat bran-enriched beverages influences gastrointestinal hormonal responses in healthy humans, J. Nutr., 2009, 139, 461-466.

47 E. Moghaddam, J. A. Vogt and T. M. S. Wolever, The effects of fat and protein on glycemic responses in nondiabetic humans vary with waist circumference, fasting plasma insulin and dietary fiber intake, J. Nutr., 2006, 136, 25062511.

48 T. M. S. Wolever, The Glycaemic Index: A Physiological Classification of Dietary Carbohydrate, CABI Publishing, Wallingford, UK, 2006, p. 66. 